\title{
FUNÇõES DA ENFERMAGEM NA VIGILÂNCIA EPIDEMIOLÓGICA *
}

\author{
Maria Jacyra de Campos Nogueira**
}

NOGUEIRA, M. J. de C. Funções da enfermagem na vigilância epidemiológica. Rev. Esc. Enf. USP, São Paulo, 15(1):29-41, 1981.

Nos países em desenvolvimento, como é o caso do Brasil, as doenças transmissíveis representam um dos principais problemas de saúde pública. Há necessidade, de acordo com a Organização Mundial de Saúde, do melhor preparo e aproveitamento da enfermeira nas ações de Vigilância Epidemiológica. Descrevem-se, no trabalho, além da conceituação de Vigilância Epidemiológica, a legislação, as atividades e o papel da enfermeira na mesma cm São Paulo.

\section{INTRODUÇÃO}

A redução da ocorrência das doenças transmissíveis ${ }^{4}$ foi uma das maiores conquistas do progresso humano neste século. Entretanto, em algumas áreas do mundo, principalmente nos países menos descnvolvidos, as doenças transmissiveis continuam a ocorrer, deixando seqüelas ou matando grande número de pessoas.

No Brasil ${ }^{1}$, clas continuam a representar as principais causas de óbito na população infantil. Além das gastroenterites e das doenças respiratórias, as doenças transmissíveis, passiveis de serem controladas pelas vacinações, continuam a atacar e a matar muitas crianças, em nosso País.

Uma das estratégias para melhorar o problema, segundo os peritos ${ }^{7}$, é aumentar a cobertura total da população pelos sistemas de serviços de saúde de cada pais ou região, utilizando, com um máximo de eficácia, os recursos disponiveis. Para essa utilização máxima, entretanto, há necessidade de reestruturação da equipe de saúde para assumir novas funções.

Um dos aspectos mais importrantes para iso é a utilização dos conceitos epidemiológicos, na prestação de serviços de saúde. A epidemiologia presta contribuições muito grandes na planificação, execução e avaliação de serviços de saúde, quer eles sejam "intra-muros" (hospitais) ou "extra-muros" (comunidade) ?

Nos paises em desenvolvimento, como é o nosso caso, há necessidade, ainda, da utilização mais racional dos recursos de enfermagem disponíveis ${ }^{7}$. Os conceitos e métodos epidemiológicos, então, têm um grande valor, em todas as fases de prestação de assistência de enfermagem, principalmente em se tratando de doenças transmissíveis.

Em todo o mundo e, também em nosso Pais, o pessoal de enfermagem sempre participou ativamente de programas de combate às doenças transmissíveis. Pelo

* Palestra proferida no Simpósio sobre Temas Atuais da Assisténcia Hospitalar e de Saúde Pública em Doenças Transmissíveis, promovido pela ABEn, Seção de São Paulo e Hospital Emilio Ribas, 1980.

* * Professor Assistente Doutor da disciplina Enfermagem Preventiva e Comunitária da EEUSP. 
cabedal de conhecimento, aliado à experiência, nesses longos anos de participação no controle de grandes endemias, na assistência a doentes hospitalizados ou não, muitas vezes em serviços improvisados, em épocas de epidemia, na educação da família, na comunidade, através das visitas domiciliárias e do atendimento na instituição de saúde, a sua responsabilidade e contribuição foi e, ainda é, de grande valia.

\section{PREVENÇÃO DAS DOENÇAS TRANSMISSIVEIS-AÇÕES DE ENFERMAGEM}

Medidas preventivas são aquelas aplicadas a individuos ou a grupos de indivíduos, quando a doença transmissivel ocorre sob forma esporádica, endêmica ou epidêmica, constituindo uma ameaça para a população.

Para que haja uma doença transmissivel, entretanto, isto é, para que o agente etiológico se perpetue, há necessidade de vários fatores (fonte ou reservatório, meio ambiente e hospedeiro ou suscetivel) denominados de "cadeia do processo infeccioso" ${ }^{10}$, a qual nada mais é que a história natural do agente etiológico animado.

$\mathrm{Na}$ prevenção das doenças transmissiveis, a enfermeira deve levar em conta todos os elementos do processo infeccioso para que este seja interrompido. Além disso, suas ações ${ }^{6}$ devem acompanhar a história natural da doença transmissível no homem ${ }^{5}$, isto é, preferivelmente iniciar-se nos indivíduos sadios, no periodo pré-patogênico, para evitar que este adquira uma infecção ou infestação (prevenção primária); quando isto não for possível, iniciar suas ações, no periodo patogênico, a fim de evitar complicações e seqüelas (prevenção secundária) e, finalmente, na impossibilidade desta atuação, assistir aos indivíduos com incapacidades ou problemas decorrentes das seqüelas, para que este se reabilite (prevenção terciária).

\section{A ENFERMAGEM NA VIGILĀNCIA EPIDEMIOLÓGICA}

\section{1 - VIGILÂNCIA EPIDEMIOLÓGICA-CONCEITO E BASES LEGAIS}

O conceito de Vigilância Epidemiológica, não é novo ${ }^{8}$. Evoluiu, de uma atitude estática, quase de policiamento, para um sentido dinâmico e mais eficiente. As ações dirigidas em sentido individual, antigas, que limitavam os movimentos de pessoas, mediante isolamento compulsório, domiciliar ou hospitalar, passaram a ser dirigidas para outra, de alerta, em relação à saúde da comunidade.

Atualmente $^{8}$ a Vigilância Epidemiológica é o estudo epidemiológico das doenças, como um processo dinâmico, que relaciona toda a cadeia do processo infeccioso; é ainda, uma atividade contínua que compreende a compilação sistemática da informação, seu estudo, análise e avaliação, destinada ao conhecimento e uso das autoridades responsáveis pelas decisões a tomar no que se refere às medidas de controle, caracterizando o denominado subsistema de informação - decisão - controle ${ }^{8}$.

A Vigilância Epidemiológica, quando bem estruturada ${ }^{8}$, dá bases para o reconhecimento e previsão das variações no comportamento das doenças transmissiveis, afim de serem tomadas medidas de controle adequadas. 
A Vigilância Epidemiológica compete, portanto ${ }^{9}$ :

- "manter um conhecimento atualizado da situação epidemiológica das doenças e dos fatores que a condicionam;

- conhecer e prever a evolução do comportamento epidemiológico das doenças;

- prever as mudanças de comportamento epidemiológico das doenças, em decorrência dos programas de controle ou erradicação;

- selecionar as medidas de controle a serem usadas".

A Vigilância Epidemiológica inclui as seguintes atividades: coleta de dados, consolidação e análise desses dados, divulgação e interpretação dos mesmos e determinação de medidas de controle.

A Vigilância Epidemiológica ${ }^{2,8,9}$ em nosso País, estruturada como é atualmen. te, iniciou-se com a lei que instituiu o Sistema Nacional de Saúde atribuindo, ao Ministério da Saúde, a Coordenação das ações relacionadas ao controle das doenças transmissíveis e responsabilidade na execução das ações relacionadas à Vigilância Epidemiológica.

\section{2 - SISTEMA NACIONAL DE VIGILÂNCIA EPIDEMIOLÓGICA}

Os elementos do Sistema Nacional de Vigilância Epidemiológica, são ${ }^{2,8}$ :

2.1. - Órgão Central, mantido no Ministério da Saúde, pela Divisão Nacional de Epidemiologia.

2.2. - Órgãos Regionais, mantidos pelas Secretarias dos Estados, Distrito Federal e Territórios.

2.3. - Órgãos Microrregionais, mantidos pelas Secretarias de Saúde dos Estados, Distrito Federal e Territórios, onde houver regionalização administrativa.

2.4. - Unidades de Vigilância Epidemiológica (UVE), que é o componente do órgão local de saúde, indicada pela respectiva Secretaria de Saúde e reconhecida pelo Ministério da Saúde. A ela estão vinculados, na sua área geográfica, os serviços de saúde, estabelecimentos de ensino, Postos de Notificação e os profissionais e ocupacionais obrigados à notificação compulsória, na qualidade de agentes de notificação.

Os laboratórios de saúde pública existentes nas áreas geográficas das UVE proporcionarão os exames complementares indicados para esclarecimentos dos diagnósticos e estarão articulados com as mesmos.

2.5. - Posto de Notificação, integrante da UVE que se limita a receber a notificação e transmitíla, imediatamente, à UVE. 


\section{3 - ÁREAS DE ATUAÇÃO DA ENFERMAGEM NA VIGILÂNCIA EPIDEMIOLÓGICA}

As ações de Vigilância Epidemiológica desenvolvem-se em diferentes áreas e com base no processo infeccioso, portanto, relacionadas às fontes de infecção, aos veículos de transmissão e à proteção dos suscetíveis ${ }^{8}$.

\section{1. - FONTES DE INFECÇÃO}

Os meios usualmente adotados para conhecimento das fontes de infeç̧ão, são descritos a seguir.

\subsection{1 - Notificação de doentes}

As informações básicas para o funcionamento do Sistema Nacional de Vigilância Epidemiológica são ${ }^{2,8}$ : notificaçōes, declarações de óbito, resultados de estudos epidemiológicos, notificações de quadros mórbidos e demais doenças julgadas de ocorrênca normal.

As doenças de notificação compulsória, em todo o território nacional, a partir de $1976^{2}$, são: febre amarela, cólera, peste, variola, hanseníase, tuberculose, poliomielite, tétano, doença, meningocócica e outras meningites, raiva humana, febre tifóide, sarampo (apenas os casos internados), leishmaniose visceral e leishmaniose cutânco-mucosa.

Em São Paulo, a Secretaria de Estado da Saúde incluiu, em $1977^{\circ}$, o seguinte elenco provisório de doenças de notificação compulsória, além daquelas determinadas ao nivel federal: difteria, doença de Chagas (forma aguda), encefalite por arbovirus, esquistossomose e malária.

Além dos médicos, no exercício de suas funções profissionais ${ }^{2,8}$, que são obrigados, particularmente, a proceder à notificação de casos suspeitos ou casos com diagnóstico confirmado, ficam obrigados a notificá-los, também, os seguintes:

- dirigentes de cada um dos estabelecimentos componentes do Sistema Nacional de Saúde que proporcionem serviços de saúde, em regime ambulatorial ou de internação;

- os dirigentes de cada um dos estabelecimentos componentes do Sistema Nacional de Saúde que executem exames complementares para diagnóstico e tratamento;

- o dirigente de estabelecimento de ensino geral, público ou particular quando lhe forem feitas comunicações através do corpo docente, pais ou responsáveis pelos alunos; tificação.

- as pessoas que exercem funções de agente de notificação, em postos de no-

As UVE devem procurar obter informações sobre casos ou óbitos e identificação dos possíveis notificantes em hospitais, clínicas, consultórios, laboratórios, farmácias, serviços médicos de empresas, cartórios de registro civil, escolas e outros locais. 
Como vemos, a enfermeira quando desempenha, principalmente, sua função administrativa em hospitais, ambulatórios e centros de saúde, dependendo de suas atribuições, colabora e/ou é responsável pela notificação compulsória de doenças expedindo ou recebendo a notificação ou orientando os serviços de saúde para o valor e a forma de executar a mesma.

\subsection{2 - Inquéritos}

Os inquéritos, como recursos utilizados na descoberta de casos ${ }^{8}$, são da maior utilidade, podendo ser empregados em população humana e animal. A enfermeira e seu pessoal auxiliar podem e devem participar dos inquéritos em população humana.

Os inquéritos podem se destinar a conhecer aspectos globais de saúde e ocorrência de doenças transmissíveis em uma localidade ou, ainda, especificamente (ex. testes imunológicos, de fezes, de sangue, etc.), para conhecer a morbidade ou nível de infecção por uma dada doença.

À enfermeira caberia, nestaárea:

- participar, conjuntamente com a equipe, no planejamento desses inquéritos, desde a formulação dos seus objetivos, atividades, até a previsão e provisão de pessoal auxiliar de enfermagem que participará dele; quéritos;

- treinar, coordenar e supervisionar o pessoal auxiliar na execução dos in-

- coordenar as atividades específicas de enfermagem;

- participar na análise e avaliação dos resultados.

\subsection{3 - Visitas domiciliárias ou em outros locais}

As visitas executadas pela enfermeira ou seu pessoal auxiliar em domicílios, escolas, locais de trabalho, além de servirem a outras finalidades dos diversos serviços das unidades sanitárias, quando têm especificamente, como objetivo prioritário, casos de doenças transmissíveis, são extremamente importantes.

Essas visitas visam primordialmente:

- conhecer a realidade bio-psico-social que está influindo no aparecimento da doença, a fim de serem tomadas medidas preventivas adequadas;

- descobrir fontes, comunicantes, casos novos e portadores a fim de serem encaminhados para receber a assistência devida;

- prestar assistência a doentes e/ou comunicantes, se for o caso;

- supervisionar os casos. 


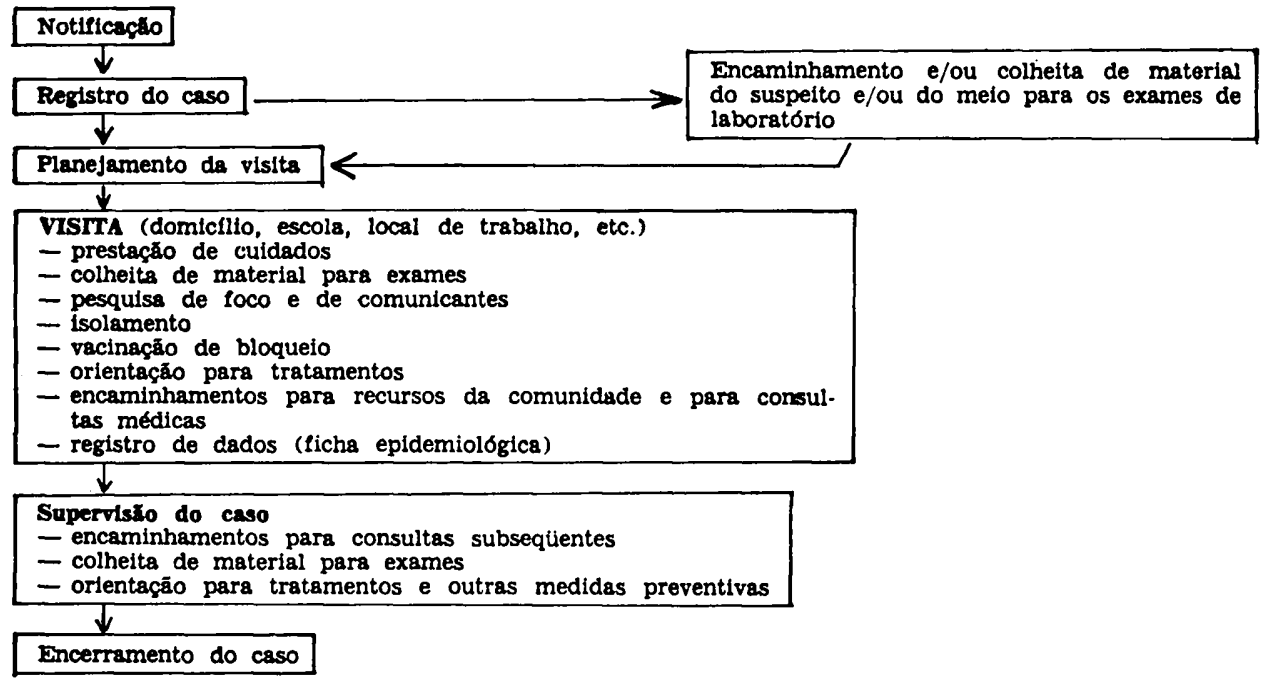

\subsection{4 - Exames de saúde}

Os exames periódicos de saúde ${ }^{8}$, como por exemplo os que são realizados para ingresso de conscritos nas Forças Armadas e outras corporações, os exame de rotina feito em escolares, etc., permitem, se bem feitos, conhecer casos ou contactos, de doenças transmissíveis, até então desapercebidos.

A supervisão de saúde realizada pela enfermeira e o atendimento de enfermagem realizado pelo pessoal de enfermagem, quando o conteúdo do levantamento de dados (histórico de enfermagem) é adequado e devidamente registrado, poderia ser uma valiosa fonte de informações para a Vigilância Epidemiológica, do mesmo modo como se constituem os exames periódicos de saúde realizados adequadamente pelo médico.

\subsection{5 - Atestados de óbito}

Os atestados de óbito, principalmente nos países em desenvolvimento, onde o número de notificações recebidas é deficiente, constituem um recurso subsidiário para o conhecimento de casos de doenças transmissiveis em uma área ${ }^{8}$.

Embora a enfermagem não tenha, diretamente, responsabilidade pelo atestado de óbito, ela deve contribuir para que este seja o mais preciso possível a fim de que sirva de base, mesmo "post-mortem", para a análise da ocorrência das doenças transmissiveis em uma região.

\subsection{6 - Controle das fontes de infecção}

Conhecida a fonte de infeç̧ão ${ }^{8}$, as providências poderão abranger os animais ou o homem. 
Cabe à enfermagem, no caso das fontes animais, a educação sanitária das famílias, principalmente em relação aos animais domésticos que podem ser fontes de infecção.

Quando se trata de fonte de infeç̧ão representada pelo homem, a assistência de enfermagem é mais ampla. Os principais aspectos são:

- isolamento hospitalar ou domiciliar;

- cuidados com fômites e descargas infectantes dos doentes;

- cuidados relativos aos tratamentos;

- educação sanitária do paciente e família.

O isolamento ${ }^{8}$ hospitalar ou domiciliar dependerá dos recursos da comunidade ou da família do paciente que representa a fonte; destina-se a estancar a fonte de infecção e, mais eficiente será quanto mais precocemente for instalado.

0 isolamento hospitalar é mais eficiente, pois, quando bem usados, são melhores. Nos domicílios é mais difícil de ser instalado e depende, não só do preparo e capacidade de improvisação do pessoal de enfermagem, como, principalmente, da colaboração e dos recursos da família.

A esterilização ou desinfecção de fômites e descargas infectantes da fonte ${ }^{8}$ (respiratórias, urinárias, intestinais, etc.) é um outro aspecto importante da assistência de enfermagem, não só no hospital como no domicílio.

A enfermeira deve conhecer e orientar o pessoal auxiliar não somente sobre os meios que podem ser usados no hospital, como também sobre àqueles que existem na comunidade e que são acessíveis à população.

Com relação aos tratamentos, um dos pontos mais importantes a serem focalizados pela enfermagem, é com relação às doses de medicamentos que visam estancar a fonte de infecção (antibióticos, quimioterápicos, etc.), pois doses e intervalos irregulares e inadequados podem torná-la resistente e fonte contínua de infecção.

A educação sanitária, enfim, é valiosíssima não só para se obter a colaboração do paciente (fonte) e sua família como, também, para a descoberta de comunicantes que, por sua vez, podem vir a ser novas fontes.

\section{2. - VEÍCULOS DE TRANSMISSÃO}

Os cuidados com os veículos de transmissão animados (seres humanos e animais) ou inanimados (água, fômtes, ar, alimentos, etc.) também são, em parte, responsabilidade do pessoal de enfermagem.

Quando o veículo é o homem ${ }^{8}$ que às vezes atua como fonte e, ao mesmo tempo, veículo de transmissão (ex.: doenças sexualmente transmitidas) os cuidados são os mesmos que e recomendam para a fonte humana e, portanto, a enfermagem tem reponsabilidade muito grande. 
As providências da enfermagem, em relação aos veículos de transmissão animal, são mais limitadas e referem-se, principalmente, à educação sanitária da população para as atividades de controle de vetores.

Com relação aos veículos inanimados ( $\mathrm{ar}$, água, alimentos, dejetos, fômites, etc.), além das ações educativas, a enfermagem tanto nos hospitais como nos domicilios, também tem responsabilidade muito grande. Valem aqui as mesmas orientações que foram ditas para as fontes de infeç̧ão.

\section{3. - PROTEÇÃO DE SUSCETIVEIS}

Os indivíduos integrantes de uma comunidade não estão igualmente expostos aos riscos de adoecerem pela mesma doença transmissivel. Portanto, as providências a serem tomadas no controle de qualquer doença transmissivel devem obedecer às várias condições prevalentes, sendo indicadas para determinados grupos etários ou especiais da comunidade que estão mais expostos aos riscos de infecção e disseminação da doença ${ }^{8}$.

Dentre as medidas para a proteção de suscetíveis ${ }^{8}$, temos o isolamento do foco, a proteção individual mecânica ou química e os preceitos de higiene individual, onde a enfermagem participa, ativamente, através da educação sanitária.

A imunização entretanto ainda é o grande recurso onde repousa a proteção dos suscetiveis ${ }^{8}$ e onde a enfermagem tem, no momento, grande responsabilidade.

Cabe à enfermeira planejar e organizar, em conjunto com a equipe, os programas e esquemas de imunização, além de treinar e supervisionar o pessoal auxiliar que aplica as vacinas, fazendo ela mesma, essa aplicação e orientando a população, em situações mais complexas.

\section{4 - PAPEL DE ENFERMAGEM NO SISTEMA DE VIGILÂCIA EPIDEMIOLÓGICA EM SÃO PAULO}

\section{1. - Sistema de Vigilância Epidemiológica - estrutura}

O Sistema de Vigilância Epidemiológica da Secretaria de Estado da Saúde, de São Paulo ${ }^{9}$, compõe-se de vários órgãos, com diferentes atribuições e que, atualmente, segundo informações, estão sendo avaliadas para que, se necessário, sejam reformuladas.

Resumidamente, a estrutura é a seguir, descrita.

NIVEL CENTRAL: Centro de Informações de Saúde (CIS)

NIVEL DE COORDENADORIAS E SUPERINTENDENCIA

- Coordenadoria de Saúde da Comunidade

Nivel Central

Nivel Regional

Nivel Distrital 
Nivel de UVE (Centro de Saúde)

Nivel de Posto de Notificação (Cenıro de Saúde)

- Coordenadoria de Assistência Hospitalar

Hospital Emílio Ribas

Outros

- Coordenadoria de Saúde Mental

- Coordenadoria de Serviços Técnicos Especializados

Nivel Central

Instituto Adolfo Lutz (Laboratório de Saúde Pública)

\section{- Superintendência de Controle de Endemias (SUCEN)}

A Secretaria de Estado da Saúde, através do CIS, para facilitar a operacionairzação das ações de Vigilância Epidemiológica, elaborou um "Manual de Vigilância Epidemiológica" 9, que contém as atribuições de cada nível, além das normas técnicas e administrativas a serem cumpridas, em um volume, para a Região da Grande São Paulo e, outro, para o restante do Estado.

Embora as atribuições estejam, também, especificadas nesses manuais, segundo as nformações ainda existem algumas distorções em relação a elas mas que pretendem corrigir, após a avaliação que vêm sendo feita.

\section{2. - Papel da enfermeira}

Nos "Padrões de Assistência de Enfermagem à Comunidade" "3, está dito que a enfermeira "participará do processo de Vigilância Epidemiológica - informação - decisão - ação, no desenvolvimento das suas atividade em todo os níveis de atuação, para o atendimento do indivíduo, família e comunidade".

De tudo o que foi dito, podemos deduzir que as tarefas principais da enfermeira, na Vigilância Epidemiológica seriam, resumidamente, as seguintes:

- coletar, processar e analisar, juntamente com a equipe, as informações básicas necesárias ao controle das doenças transmissíveis; trole;

- elaborar juntamente com essa equipe, programas de ação para o seu con-

- coletar, processar e analisar os dados referentes às necessidades e os recursos disponiveis de enfermagem para esses programas;

- planejar, organizar, coordenar e supervisionar os serviços e/ou atividades de enfermagem desses programas;

- planejar, executar e avaliar programas de treinamento de pessoal auxiliar de enfermagem que atuará nesses programas; 
- supervisionar a população sob risco, através da assistência de enfermagem dada nas consultas e atendimentos de enfermagem, visitas domiciliárias, vacinação, etc.;

- supervisionar a assistência de enfermagem prestada pelo pessoal auxiliar de enfermagem nas unidades sanitárias ou na comunidade, na execução das ações de Vigilância Epidemiológica;

- executar essas atividades quando o grau de complexidade for maior e/ou quando necessário;

- educar individualmente e/ou grupo, a respeito da prevenção das doenças transmissiveis;

- planejar, executar e participar de pesquisas epidemiológicas.

Com a finalidade de verificar quais as atribuições de vigilância epidemiológica das enfermeiras de São Paulo, para subsidiar o ensino de graduação de enfermagem ou reformulação nas atribuições, se necessário, realizamos um levantamento junto às enfermeiras da Coordenadoria de Saúde da Comunidade, na Gran. de São Paulo, em novembro de 1980.

Os objetivos desse levantamento foram:

- verificar quais eram as atribuições da enfermeira na Vigilância Epidemiológica, ao Nível Central, Regional, Distrital e Local;

- verificar se existem problemas decorrentes dessas atribuições;

- verificar quais seriam as sugestões para resolver os problemas que, porventura, existissem.

Para a coleta de informações, foram entrevistadas as seguintes enfermeiras: uma ao Nível Central, uma ao Nível Regional (9,6\%); duas ao Nivel Distrital $(10,0 \%)$ e quatro ao Nivel Local $(20,0 \%)$.

Os resultados, resumidamente, foram os seguintes: as enfermeiras que estão localizadas no Nível Central, Regional e Distrital da Coordenadoria de Saúde da Comunidade, na Região da Grande São Paulo, informaram que têm pouco ou quase nenhuma atribuição nas açōes de Vigilância Epidemiológica. São, esporadicamente, convidadas a opinar apenas quando, nos niveis de planejamento, há necessidade de assessoria de enfermagem.

Ao nível de UVE, a participação é bem maior pois praticamente quase todas as ações de Vigilância Epidemiológica são atribuições do pessoal de enfermagem. Essas atividades, ora executadas pela enfermeira (as mais complexas), ora executadas pela visitadora (as mais simples), resumem-se em: receber e registrar a notificação, abrir a ficha epidemiológica especifica para cada doença, planejar e executar as visitas, proceder à vacinação de bloqueio, encaminhar os clientes para exames de laboratório ou consulta médica. As enfermeiras, além da supervisão do pessoal auxiliar que executa essas atividades, entram em contacto com os hospitais e outras agências obrigadas a fazer a notificação, para com estes manter bom entrosamento e colher os dados de notificação. 
Os principais problemas ou dificuldades que as enfermeiras das UVE estão encontrando, segundo as mesmas, são as seguintes:

- algumas enfermeiras das UVE não recebem assessoria dos Niveis Distrital e Regional pois os mesmo não estão envolvidos nas açōes de Vigilância Epidemiológica, como deveriam estar. Em caso de dúvida os problemas são resolvidos por consulta a individuos mais experientes e de boa vontade.

- falta pessoal (visitadoras) para todas as visitas necessárias. A enfermeiras das UVE, que se localizam nos centros de saúde maiores, acumulam outras atribuições e não só as de vigilância epidemiológica $e$, portanto, não conseguem dispor de tempo suficiente para a vigilância epidemiológica adequadamente; algumas chegam a levar para casa muitas atividades de registro de dados para fazer, após o expediente, o serviço do centro de saúde;

- faltam recursos materiais para execução das ações de vigilância epidemiológica, principalmente transporte; os veículos dos centros de saúde onde estão localizadas as UVE, não estão disponiveis somente para vigilância epidemiológica e, portanto, a atividade de visita fica difícil, quando não impossível de ser realizada;

- as enfermeiras não foram convidadas, na época da implantação das UVE, para as reuniōes de treinamento, como foi feito para com os médicos sanitaristas, sendo que algumas, prevendo as dificuldades futuras, participaram dessas reuniōes, por iniciativa própria; há, pois, problemas referentes ao treinamento e supervisão do pessoal auxiliar que ficou sob sua responsabilidade; este sente que as enfermeiras dos níveis superiores não participaram, tampouco, do planejamento do Sistema de Vigilância Epidemiológica e que, no momento têm que executar atividades em cujo planejamento não puderam participar; daí as dificuldades que estão encontrando;

- além da visitadora, falta escriturário para executar tarefas mais simples de registro de dados, sendo que o pessoal de enfermagem é desviado de outras ativiades próprias e necessárias para acumular esta atribuição;

- a comunicação, em alguns locais distantes da Região da Grande São Paulo, ainda é muito difícil pois nem telefone algumas UVE têm;

- as fichas epidemiológicas, segundo algumas enfermeiras, são muito detalhadas e não há pessoal suficiente para preenche-las; deveriam ser simplificadas ou então deveria haver maior número de pessoal para preenche-las;

- não existem, por escrito, as atribuições de cada categoria profissional, na Vigilância Epidemiológica, inclusive do pessoal de enfermagem; isto é, em alguns casos, tem dificultado a divisão do trabalho e a sobrecarga do pessoal de enfermagem;

- há dificuldade e gasto de tempo muito grande, na consulta dos prontuários dos hospitais, por ocasião da coleta de informações para a notificação, pois os prontuários são prolixos, têm muitas folhas e as letras nem sempre são legíveis.

As sugestões dadas pelas enfermeiras das UVE, para que esses problemas sejam sanados, são as seguintes: 
- formulação de atribuições da equipe e de pessoal de enfermagem, em todos os níveis, na Vigilância Epidemiológica, incluindo maior participação da enfermeira no planejamento e não só na execução das ações de vigilância;

- planejamento e execução de programas de treinamento e atualização do pessoal de enfermagem em Vigilância Epidemiológica;

- simplificação das normas de registro de dados;

- entrosamento melhor com os hospitais, para a melhoria dos prontuários; tritais;

- descentralização das atividades de Vigilância aos Níveis Regionais e Dis-

- provimento dos centros de saúde de recursos humanos e materiais suficientes, solicitados pelas enfermeiras;

- elaboração de um sistema que permita o retorno, aos Níveis Regionais, Distritais e Locais, das informações por estes enviadas ao CIS e lá consolidadas; a finalidade seria para que a equipe tomasse conhecimento da situação de saúde de sua região e assim tivesse condições de avaliá-la, tomar providências e fazer estudo epidemiológicos.

\section{CONCLUSŌES}

As doenças transmissíveis, no Brasil, representam um problema de Saúde Pública. A Vigilância Epidemiológica visa contribuir para a melhoria desse problema. A enfermeira de Saúde pública e seu pessoal auxilar têm um papel relevante nas ações de Vigilância Epidemiológica e há necessidade de um estudo para restruturação das atribuições desses elementos para que sejam mais bem aproveitados.

As enfermeiras nos Niveis Central, Regional e Distrital, da Coordenadoria de Saúde da Comunidade, da Secretaria de Estado da Saúde, na Grande São Paulo, praticamente não têm atribuições na Vigilância Epidemiológica. A Nivel Local (UVE) as atribuições, embora não existam formuladas por escrito, são aquelas preconizadas pela Vigilância Epidemiológica. A execução, entretanto, é feita com muita dificuldade, principalmente por não tercm as enfermeiras participado do planejamento, por não ter o pessoal de enfermagem sido treinado suficientcmente e por inexistirem recursos humanos e materiais suficientes.

As principais sugestões para a melhoria desses problemas são referentes à elaboração de atribuições, descentralização adquada de ações, previsão e provisão de recursos e treinamento do pessoal para que, segundo a Organização Mundial de Saúde e os "Padrões de Assistência à Comunidade", a enfermeira possa participar mais efetivamente na cobertura das ações de Vigilância Epidemiológica.

\section{AGRADECIMENTOS}

Ao Dr. José de Souza Moraes, à Enfermeira Ester Barros Domingues e Dra. Maria Inez Batistella, do Departamento Regional da Grande São Paulo, da Coordenadoria de Saúde da Comunidade da Secretaria de Estado da Saúde, pelas informações prestadas e pela autorização para as entrevistas. 
As Enfermeiras da Coordenadoria de Saúde da Comunidade, da Região da Grande São Paulo, entrevistadas, pela colaboração.

NOGUEIRA, M. J. de C. The nursing in epidemiological vigilance. Rev. Esc. Enf. USP, São Paulo, 15(1):29-41, 1981.

In developing contries, transmissible diseases area a public health problem. According to the World Health Organization, increasing utilization of nurses in Epidemiological Vigi. lance actions is necessary. The author describes concepts, legislation and nursing activities in Great São Paulo, Brasil, in Epidemiological Vigilance.

\section{REFERENCIAS BIBLIOGRAFICAS}

1. BRASIL. Ministério da Saúde. Sistema Nacional de vigilância epidemiológica. In:

Anais da 5.a Conferência Nacional de Saúde, Brasília, 5-8 agosto 1975. Brasilia, 1975. p. 137-48.

2. BRASIL. Secretaria Nacional de Ações Básicas e Saúde. Vigilância epidemiológica e imunizações básicas: legislação básica. 3. ed. Brasília, 1977. $36 \mathrm{p}$.

3. BRASIL. Ministério da Saúde. Padrões de assistência de enfermagem à comunidade. Brasilia, 1976/1979. $71 \mathrm{p}$.

4. FORATTINI, O. P. Epidemiologia geral. São Paulo, Edgar Blücher, EDUSP, 1976. 259 p.

5. LEAVELL, H. \& CLARK, G. Medicina preventiva. São Paulo, McGraw.Hill, 1976. 744 p.

6. NOGUEIRA, M. J. de C. Enfermagem preventiva. Enf. Novas Dimens., São Paulo, 5(3): 161-2, maio/jun. 1979.

7. ORGANIZACION PANAMERICANA DE LA SALUD. La epidemiologia y la enfermera. Washington, 1976. 12 p. (Publicación Cientifica, 333).

8. RODRIGUES, B. A. Fundamentos de administração sanitária. 2. ed. Brasilia, Centro Gráfico do Senado Federal, 1979. $387 \mathrm{p}$.

9. SAO PAULO (Estado). Secretaria da Saúde. Manual de vigilância epidemiológica. São Paulo, 1977.

10. Universidade de São Paulo. Faculdade de Saúde Pública. Departamento de Epidemiologia. Postila de epidemiologia. São Paulo, s.d. (mimeografado). 